\title{
INTRODUCIENDO LA DIMENSIÓN MOTORA DENTRO DE LA CONCEPTUALIZACIÓN DE LA EXPERIENCIA DEL DOLOR
}

\author{
Roy La Touche, $\mathrm{PhD}^{1,2,3}$ \\ 1. Departamento de Fisioterapia, Facultad de Ciencias de la Salud. Centro Superior de Estudios Universitarios La Salle, Universidad Autónoma de Madrid, \\ Madrid, España. \\ 2. Motion in Brains Research Group, Instituto de Neurociencias y Ciencias del Movimiento (INCIMOV), Centro Superior de Estudios Universitarios La Salle, \\ Universidad Autónoma de Madrid, Madrid, España. \\ 3. Instituto de Neurociencia y Dolor Craneofacial (INDCRAN), Madrid, España.
}

Correspondencia:

Roy La Touche, PT, PhD. Departamento de Fisioterapia. CSEU La Salle. Universidad Autónoma de Madrid. Calle La Salle, $n^{\circ}$ 10, 28023 Madrid, España Teléfono: (+34) 917401980

Fax: (+34) 913571730 E-mail: Roylatouche@yahoo.es

DOI:

https://doi.org/10.37382/jomts.v3i1.474
La conceptualización del dolor históricamente ha tenido diversas interpretaciones que van desde la perspectivas más filosóficas o antropológicas hasta las orientaciones científico-clínicas. Es posible que la evolución más importante en este campo haya sido el abandonar la visión del dolor como un mecanismo absolutamente sensitivo a plantearlo como una experiencia multidimensional.

Inicialmente la propuesta más predominante y extendida formalmente fue la que establecía una bidimensionalidad de la experiencia dolorosa que abarcaba la dimensión sensorial discriminativa y la afectiva motivacional (Price, 2000; Auvray et al., 2010). La Asociación Internacional para el Estudio del Dolor asumió esta bidimensionalidad al definir el dolor como "una experiencia sensorial y emocional desagradable asociada o similar a la asociada con daño tisular real o potencial" (Raja et al., 2020; IASP, 2021).

Aunque el motivo de este escrito no es realizar una crítica a esta definición, es muy importante reconocer que el dolor como marcador biológico fiable de daño tisular, a la luz de la evidencia actual, es muy cuestionable (Auvray et al., 2010; Moseley and Vlaeyen, 2015) y está más cerca de interpretarse como una experiencia que influye sobre la conducta. A pesar del conocimiento y los avances científicos actuales sobre el dolor, la organización científica más importante en el mundo en términos conceptuales de dolor sigue una realidad continuista de más de 4 décadas, y el consenso para el cambio de definición parece imposible (Turner and ArendtNielsen, 2020).

\section{El Concepto del Dolor}

Atendiendo a la evidencia científica actual y centrando la idea de este breve planteamiento teórico propongo la siguiente definición que va a servir de enlace para operativizar y organizar los siguientes extractos del argumento que se va a desarrollar a lo largo del texto: "el dolor es una compleja experiencia multidimensional de carácter perceptivo dependiente del contexto y los procesos evaluativos individuales que influye significativamente sobre la conducta". 
En esta definición asumimos el legado teórico contemporáneo que considera el dolor como una experiencia multidimensional que abarca componentes sensoriales, cognitivos, emocionales y considero que es de vital importancia el incluir la dimensión motora. En esta propuesta conceptual se incluye la percepción como el mecanismo central del procesamiento de información; este proceso está directamente influido por el contexto, (constituye parte del dominio social) y la evaluación individual de la experiencia, este último punto es importante ya que se le otorga una amplia importancia al procesamiento cognitivo que incluiría los mecanismos de aprendizaje, las experiencias pasadas, las expectativas y la toma de decisiones. Los elementos anteriormente citados de forma individual o en conjunto deben de ser considerados como condicionantes de conducta ante la experiencia dolorosa. Algunas de las respuestas conductuales muy habituales encontradas ante la experiencia dolorosa son las derivadas del comportamiento motor.

\section{La Experiencia Multidimensional del Dolor}

Ronald Melzack desarrolló la teoría de la neuromatriz del dolor en donde ampliaba la visión de la experiencia de dolor integrando aspectos más dinámicos como la inhibición, la excitación y la modulación que se producen a través de una amplia red neuronal que implica funciones cognitivas, sensoriales y afectivas (Melzack, 1999), básicamente Melzack intentó darle un sustrato neurofisiológico cortical y subcortical a la experiencia de dolor que sirviera como marco de trabajo para desarrollar las propuestas de diagnóstico y de tratamiento. La teoría de la neuromatriz del dolor adquirió mucha relevancia clínica en términos diagnósticos y terapéuticos ya que otorga la misma importancia a los aspectos sensoriales, afectivos y cognitivos de la experiencia de dolor (Cárdenas Fernández, 2015).

La teoría de Melzack se postula dentro de una perspectiva multidimensional que involucra las siguientes dimensiones (Melzack, 1999):

- Dimensión sensorial-discriminativa: incluye las características espaciales, temporales y la cualidad de la experiencia dolorosa (Auvray et al., 2010).

- Dimensión motivacional-afectiva: comporta todos los aspectos emocionales relacionados con la experiencia dolorosa.

- Dimensión cognitivo-evaluativa: son los procesos involucrados en el análisis e interpretación de la experiencia dolorosa en función de la percepción sensorial y la predicción de los cambios de conducta que pueden ocurrir (Melzack and Torgerson, 1971).

Las dimensiones de la experiencia dolorosa son útiles como marco teórico para el desarrollo de propuestas de investigación o para los procesos clínicos que integran el razonamiento clínico y la toma de decisiones, sin embargo hay que evitar pensar en las dimensiones como elementos individuales, las dimensiones son fundamentalmente un sistema de interacción mutuo, entendiendo que en determinas ocasiones alguna dimensión adquiere mayor relevancia, sin que esto implique la no participación de las demás. La propuesta que se presenta en este escrito es tretradimensional, incluye todas las dimensiones descritas anteriormente más la dimensión del comportamiento motor (Figura 1).

\section{La Dimensión del Comportamiento Motor}

Las alteraciones del movimiento relacionadas con el dolor implican neurofisiológicamente mecanismos centrales y periféricos que presentan diversos niveles o grados de influencia que determinan el desempeño conductual (Holmes et al., 2021). En las últimas dos décadas se han desarrollado diversas teorías que intentan explicar la relación entre el dolor y el movimiento (Merkle et al., 2020), estas teoría están diseñadas a partir de los hallazgos obtenidos en estudios de dolor inducido experimentalmente (Graven-Nielsen et al., 2009) o de investigaciones en donde evalúan los déficits funcionales o la discapacidad a través de la influencia o interacción de componentes sensoriales, cognitivos, emocionales y motores en pacientes con dolor de larga evolución (Picavet et al., 2002; Sullivan et al., 2009; George et 
al., 2011; Lambin et al., 2011; La Touche et al., 2019). elemento más dentro del concepto de experiencia multidimensional del dolor. El comportamiento motor conceptualmente incluye todo tipo

Figura 1. Representación gráfica del concepto multidimensional de la experiencia del dolor.

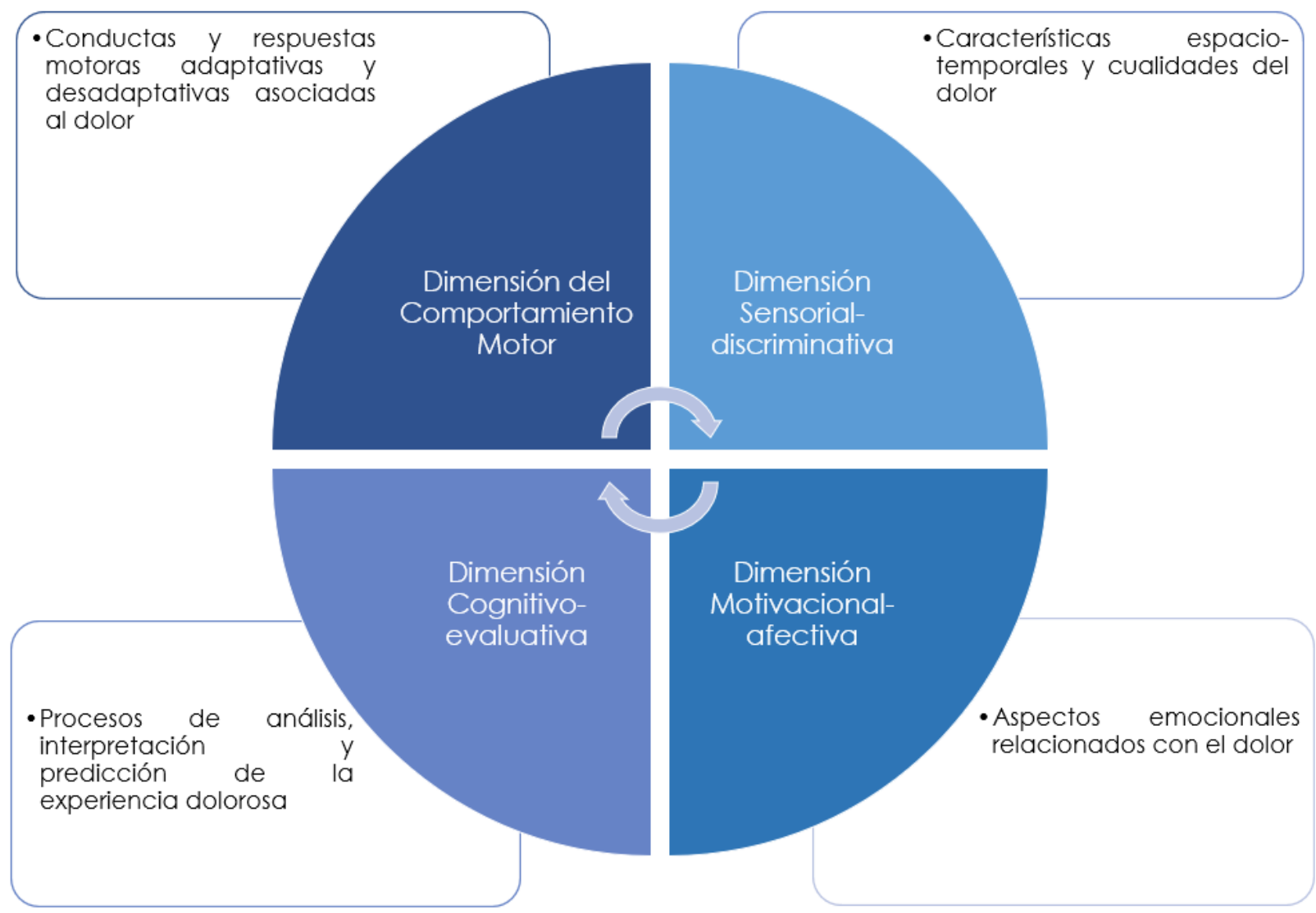

El concepto de experiencia multidimensional de dolor está sustentado por la evidencia científica actual, sin embargo los componentes motores (dimensión motora) implicados en la experiencia dolorosa han sido simplificados, excluidos o minimizados (Butera et al., 2016) a pesar de que las alteraciones funcionales, los bajos niveles de actividad física y la discapacidad son de las principales alteraciones que presentan los pacientes con dolor, sobre todo los de larga evolución. Podemos afirmar que las alteraciones derivadas del movimiento son las principales consecuencias disfuncionales que nos encontramos en los pacientes que presentan dolores de larga evolución.

Desde el punto de vista científico, pero sobre todo desde la visión clínica resulta indispensable añadir la dimensión del "comportamiento motor" como un movimiento, desde las respuestas motoras involuntarias, hasta las acciones dirigidas a un objetivo concreto desarrollado en todos los contextos físicos y sociales (Adolph and Franchak, 2017).

La dimensión del comportamiento motor se establece a partir de la premisa general de que el dolor genera cambios en la forma en que nos movemos, y la forma en que nos movemos puede cambiar la forma en que experimentamos $y$ percibimos la experiencia dolorosa (Hodges and Tucker, 2011; Bank et al., 2013; Paris et al., 2013). De forma más concreta algunos autores sugieren que el movimiento está involucrado en la experiencia dolorosa inicialmente como una respuesta adaptativa y protectora para controlar o disminuir la percepción de dolor (Hodges and Smeets, 2015; Merkle et al., 2020), estas respuestas evocadas pueden influir sobre 
el movimiento alterando la velocidad, la variabilidad y la eficiencia neuromuscular.

Simmonds et al, consideran que las respuestas del movimiento no son únicamente una consecuencia de anticipar y minimizar el dolor, propone que el comportamiento motor implicado en el dolor es un factor muy complejo de analizar; los factores psicológicos (cogniciones y emociones), sociales y contextuales pueden influir sobre la actividad motora dentro del componente conductual del dolor como experiencia multidimensional (Simmonds et al., 2008).

Con respecto a la función protectora del movimiento relacionado con el dolor, cabe mencionar que cuando no es necesaria o biológicamente no está justificada y se mantiene esta función, las adaptaciones motoras que se presentan en la mayoría de los casos se consideran desadaptivas e involucran cambios del comportamiento motor que afectan la función, el aprendizaje motor, la gestión, la planificación del movimiento, capacidades físicas concretas. Además, en algunos casos puede presentarse un incremento de las conductas de evitación que surgen a través de la interacción con factores contextuales, cognitivos y afectivosmotivacionales y que incrementan el nivel de discapacidad.

Otro aspecto que se debe de tomar en consideración es que el comportamiento motor como elemento esencial de la experiencia dolorosa puede presentarse a través de múltiples respuestas, por ejemplo, en pacientes con dolor disfuncional de larga evolución puede darse el caso que se utilicen estrategias pasivas de movimiento para evitar el dolor, sin embargo otros pacientes utilizan estrategias activas de auto-regulación para hacer frente al dolor (Van Damme and Kindermans, 2015).

Finalmente definimos la dimensión del comportamiento motor como el conjunto de las respuestas motoras adaptativas o desadaptativas relacionados con la experiencia dolorosa que afectan la modulación, el procesamiento y la función, y que además interactúan o se ven influenciados por factores contextuales, cognitivos y afectivosmotivacionales (Figura 2).

\section{El Sustrato Neural de la Dimensión del Comportamiento Motor}

La teoría de la neuromatriz del dolor le otorgó a las dimensiones de la experiencia dolorosa un fundamento morfo-fisiológico de las áreas del sistema nervioso central relacionados con el procesamiento y la modulación del dolor (Melzack, 1999), evidencia científica basada en análisis metaanalíticos de estudios de neuroimagen han refrendado la participación de áreas anatómicas concretas del sistema nervioso central asociadas a la dimensiones sensorial, cognitiva y afectiva del dolor (Peyron et al., 2000). La investigación sobre el sustrato neural relacionado con la experiencia dolorosa y el comportamiento motor genera mucho interés en la actualidad.

Hallazgos de investigación básica de carácter anatómico describen la existencia de proyecciones nociceptivas directas del tracto espinotalámico hacia áreas motoras (Dum et al., 2009), por otra parte, existe evidencia que sugiere que los correlatos neurales implicados en la experiencia dolorosa incluyen la actividad relacionada con los procesos perceptivos, los motores y la actividad de sistema nervioso autónomo (Piché et al., 2010; Tiemann et al., 2018; Yeater et al., 2021). Otros autores describen, que los estímulos nociceptivos mantenidos pueden evocar una inhibición de la corteza motora generando alteraciones en la actividad neuromuscular, este proceso puede incrementar la actividad del sistema nervioso simpático y alterar el funcionamiento de vías aferentes (propiocepción, procesamiento somatosensorial) implicadas en el movimiento (Nijs et al., 2012).

A pesar de la creciente cantidad de estudios sobre las interacciones entre el dolor y la actividad encefálica asociada al comportamiento motor aún no se tiene completamente claro la especificidad y relevancia de estas respuestas neurales (Postorino et al., 2017), otro problema es que en la literatura actual referente a la activación de la corteza motora primaria en los casos de dolor crónico es contradictoria en diversos aspectos (Wei-Ju et al., 2018). Hoy en día podemos decir sobre este tema que tenemos un conjunto de teorías adecuadamente 
justificadas con múltiples incógnitas por resolver, por eso es muy necesario seguir realizando más investigación en esta línea. que además presentan catastrofismo ante el dolor específicamente el sub-constructo de desesperanza. (Salomons et al., 2012).

Es importante destacar que en la evidencia

Figura 2. El papel de la dimensión del comportamiento motor dentro de la experiencia multidimensional del dolor.

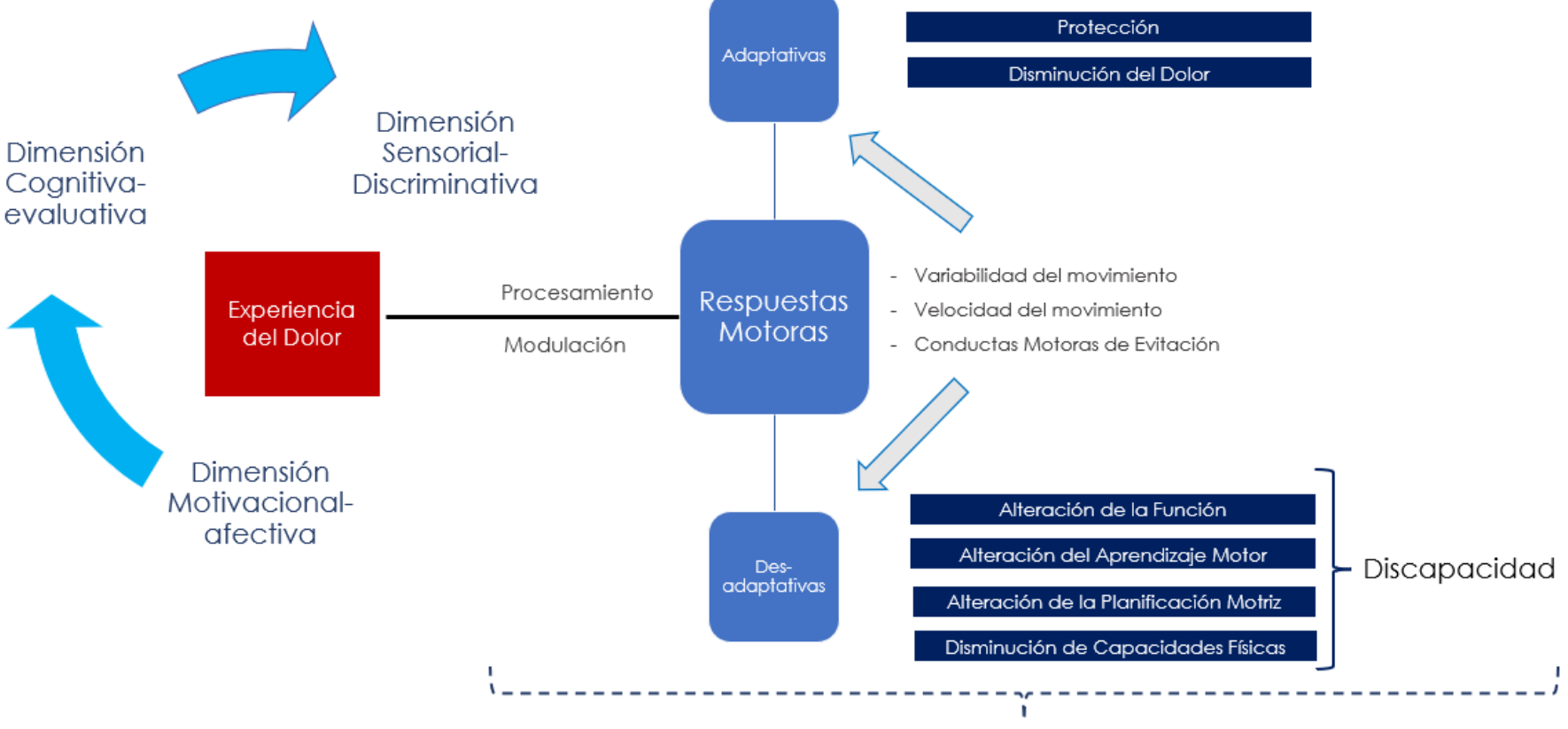

Dimensión del Comportamiento Motor

Evidencia derivada de estudios de neuroimagen indican que el procesamiento de la experiencia dolorosa evoca la activación de áreas relacionados con la gestión y planificación de la actividad motora como son la corteza motora primaria, el área premotora, el área motora suplementaria y la corteza cingulada anterior (Gelnar et al., 1999; Perini et al., 2013; Wager et al., 2013; Misra and Coombes, 2015), en relación a esto, en pacientes con síndrome de dolor regional complejo se ha observado una reorganización de circuitos motores mostrando una mayor activación de la corteza motora primaria y el área motora suplementaria, además, estos patrones neurofisiológicos se correlacionaron con el grado de disfunción motora (Maihöfner et al., 2007). Sobre los cambios estructurales en áreas motoras, se han reportado cambios en el grosor cortical del área motora suplementaria en pacientes con dolor crónico científica reciente se ha demostrado que la corteza cingulada anterior y el área motora suplementaria presentan una actividad superpuesta en el procesamiento de la experiencia dolorosa y en el control motor (Misra and Coombes, 2015). Otras áreas que se han visto involucradas en la percepción de dolor y en el comportamiento motor son el cerebelo (Coombes and Misra, 2016) y el putamen (Azqueta-Gavaldon et al., 2020).

\section{La Modulación del Dolor a través del Sistema Motor}

La modulación del dolor a través del sistema motor consiste fundamentalmente en el proceso de activación directa o indirecta de procesos relacionados con la gestión, planificación y producción del movimiento para disminuir la percepción de la experiencia dolorosa (Holmes et al., 
2021). Es acertado suponer desde los hallazgos de evidencia disponible que estos procesos pueden estar influenciados por variables cognitivas, emocionales o contextuales que a su vez puedan favorecer o condicionar la modulación del dolor relacionado con el sistema motor.

Los mecanismos neurofisiológicos relacionados con la modulación del dolor y su asociación con la activación del sistema motor son variados y muchos de ellos están aún por definirse (Holmes et al., 2021). Sin embargo, las modalidades por las que se puede evocar este tipo de modulación han sido investigados en múltiples estudios y entre ellos se encuentran:

- Las respuestas motoras evocadas en el dolor agudo como proceso adaptativo para disminuir el dolor.

- Diversas modalidades de ejercicio terapéutico.

- Los métodos de representación del movimiento (imaginería motora, entrenamiento de observación de acciones y la terapia de espejo).

- Las técnicas de neuromodulación de la corteza cerebral que además en muchos de los casos se dirigen a la estimulación de la corteza motora primaria (a. Estimulación magnética transcraneal; b. Estimulación transcraneal de corriente continua; c. Estimulación cerebral profunda de la corteza motora).

\section{Notas Finales}

En este planteamiento teórico se propone un diseño y re-conceptualización de la experiencia dolorosa manteniendo el enfoque multidimensional, pero introduciendo una dimensión más, que se denomina "comportamiento motor".

La dimensión del comportamiento motor incluye todas los procesos y respuestas asociadas a la experiencia dolorosa que pueden manifestarse de forma adaptativa o desadaptativa y que influyen de forma directa sobre el procesamiento y la modulación del dolor y además interactúan de forma circundante con los procesos afectivos-motivacionales, los sensoriales y los cognitivos.

\section{REFERENCIAS}

Adolph KE, Franchak JM. The development of motor behavior. Wiley Interdiscip Rev Cogn Sci. 2017;8(1-2) DOI: http://dx.doi.org/10.1002/WCS.1430.

Auvray M, Myin E, Spence C. The sensory-discriminative and affective-motivational aspects of pain. Neurosci Biobehav Rev. Pergamon; 2010;34(2):214-23 DOI: http://dx.doi.org/10.1016/J.NEUBIOREV.2008.07.008.

Azqueta-Gavaldon M, Youssef A, Storz C, Lemme J, SchulteGöcking H, Becerra L, Azad S, Reiners A, Ertl-Wagner B, D B, J U, E K. Implications of the putamen in pain and motor deficits in complex regional pain syndrome. Pain. 2020;161(3):595-608 DOI: http://dx.doi.org/10.1097/J.PAIN.0000000000001745.

Bank P, Peper C, Marinus J, Beek P, van Hilten J. Motor consequences of experimentally induced limb pain: a systematic review. Eur J Pain. 2013;17(2):145-57 DOI: http://dx.doi.org/10.1002/J.1532-2149.2012.00186.X.

Butera KA, Fox EJ, George SZ. Toward a Transformed Understanding: From Pain and Movement to Pain With Movement. Phys Ther. 2016;96(10):1503 DOI: http://dx.doi.org/10.2522/PTJ.20160211.

Cárdenas Fernández R. La neuromatrix y su importancia en la neurobiologia del dolor. Invest Clin. 2015;56(2):109-10.

Coombes S, Misra G. Pain and motor processing in the human cerebellum. Pain. 2016;157(1):117-27 DOI: http://dx.doi.org/10.1097/J.PAIN.0000000000000337.

Van Damme S, Kindermans H. A self-regulation perspective on avoidance and persistence behavior in chronic pain: new theories, new challenges? Clin J Pain. 2015;31(2):115-22 DOI: http://dx.doi.org/10.1097/AJP.0000000000000096.

Dum RP, Levinthal DJ, Strick PL. The Spinothalamic System Targets Motor and Sensory Areas in the Cerebral Cortex of Monkeys. J Neurosci. 2009;29(45):14223 DOI: http://dx.doi.org/10.1523/JNEUROSCI.3398-09.2009.

Gelnar P, Krauss B, Sheehe P, Szeverenyi N, Apkarian A. A comparative fMRI study of cortical representations for thermal painful, vibrotactile, and motor performance tasks. Neuroimage. 1999;10(4):460-82 DOI: http://dx.doi.org/10.1006/NIMG.1999.0482.

George SZ, Calley D, Valencia C, Beneciuk JM. Clinical Investigation of Pain-related Fear and Pain Catastrophizing for Patients With Low Back Pain. Clin J Pain. 2011;27(2):108-15 DOI: http://dx.doi.org/10.1097/AJP.0b013e3181f21414.

Graven-Nielsen T, SVENSSON P, ARENDT-NIELSEN L. Effect of Muscle Pain on Motor Control: A Human Experimental Approach. http://dx.doi.org/101080/140381900443418 [Internet]. 2009 [cited 2021 Sep 8];2(1):26-38 DOI: http://dx.doi.org/10.1080/140381900443418. Available from:

https://www.tandfonline.com/doi/abs/10.1080/1403819004 43418

Hodges P, Smeets S. Interaction between pain, movement, and 
physical activity: short-term benefits, long-term consequences, and targets for treatment. Clin J Pain. 2015;31(2):97-107

DOI: http://dx.doi.org/10.1097/AJP.0000000000000098.

Hodges PW, Tucker K. Moving differently in pain: A new theory to explain the adaptation to pain. Pain. 2011;152(Supplement):S90-8 DOI: http://dx.doi.org/10.1016/j.pain.2010.10.020.

Holmes S, Kim A, Borsook D. The brain and behavioral correlates of motor-related analgesia (MRA). Neurobiol Dis. 2021;148:105158 DOI: http://dx.doi.org/10.1016/J.NBD.2020.105158.

IASP. Terminology | International Association for the Study of Pain. 2021.

Lambin D, Thibault P, Simmonds M, Lariviere C, Sullivan M. Repetition-induced activity-related summation of pain in patients with fibromyalgia. Pain. 2011;152(6):1424-30 DOI: http://dx.doi.org/10.1016/J.PAIN.2011.02.030.

Maihöfner C, Baron R, DeCol R, Binder A, Birklein F, Deuschl G, Handwerker HO, Schattschneider J. The motor system shows adaptive changes in complex regional pain syndrome. Brain. 2007;130(10):2671-87 DOI: http://dx.doi.org/10.1093/brain/awm131.

Melzack R. From the gate to the neuromatrix. Pain. 1999;Suppl 6:S121-6.

Melzack R, Torgerson W. On the language of pain. Anesthesiology. 1971;34(1):50-9 DOI: http://dx.doi.org/10.1097/00000542-197101000-00017.

Merkle S, Sluka K, Frey-Law. L. The interaction between pain and movement. J Hand Ther. 2020;33(1):60-6 DOI: http://dx.doi.org/10.1016/J.JHT.2018.05.001.

Misra G, Coombes S. Neuroimaging Evidence of Motor Control and Pain Processing in the Human Midcingulate Cortex. Cereb Cortex. 2015;25(7):1906-19 DOI: http://dx.doi.org/10.1093/CERCOR/BHU001.

Moseley G, Vlaeyen J. Beyond nociception: the imprecision hypothesis of chronic pain. Pain. 2015;156(1):35-8 DOI: http://dx.doi.org/10.1016/J.PAIN.0000000000000014.

Nijs J, Daenen L, Cras P, Struyf F, Roussel N, Oostendorp RAB. Nociception affects motor output: A review on sensorymotor interaction with focus on clinical implications. Clin J Pain [Internet]. Clin J Pain; 2012 [cited 2020 Nov 2];28(2):175-81

DOI: http://dx.doi.org/10.1097/AJP.0b013e318225daf3.

Available https://pubmed.ncbi.nlm.nih.gov/21712714/

from:

Paris T, Misra G, Archer D, Coombes S. Effects of a force production task and a working memory task on pain perception. J pain. 2013;14(11):1492-501 DOI: http://dx.doi.org/10.1016/J.JPAIN.2013.07.012.

Perini I, Bergstrand S, Morrison R. Where pain meets action in the human brain. J Neurosci. 2013;33(40):15930-9 DOI: http://dx.doi.org/10.1523/JNEUROSCI.3135-12.2013.

Peyron R, Laurent B, García-Larrea L. Functional imaging of brain responses to pain. A review and meta-analysis (2000). Neurophysiol Clin. 2000;30(5):263-88 DOI:
http://dx.doi.org/10.1016/S0987-7053(00)00227-6.

Picavet H, Vlaeyen J, Schouten J. Pain catastrophizing and kinesiophobia: predictors of chronic low back pain. Am J Epidemiol. 2002;156(11):1028-34 DOI: http://dx.doi.org/10.1093/AJE/KWF136.

Piché M, Arsenault M, Rainville P. Dissection of perceptual, motor and autonomic components of brain activity evoked by noxious stimulation. Pain. 2010;149(3):453-62 DOI: http://dx.doi.org/10.1016/J.PAIN.2010.01.005.

Postorino M, May ES, Nickel MM, Tiemann L, Ploner M. Sensory Processing: Influence of pain on motor preparation in the human brain. J Neurophysiol. 2017;118(4):2267 DOI: http://dx.doi.org/10.1152/JN.00489.2017.

Price D. Psychological and neural mechanisms of the affective dimension of pain. Science. 2000;288(5472):1769-72 DOI: http://dx.doi.org/10.1126/SCIENCE.288.5472.1769.

Raja SN, Carr DB, Cohen M, Finnerup NB, Flor H, Gibson S, Keefe FJ, Mogil JS, Ringkamp M, Sluka KA, Song XJ, Stevens B, Sullivan MD, Tutelman PR, Ushida T, Vader K. The revised International Association for the Study of Pain definition of pain: concepts, challenges, and compromises. Pain. 2020;161(9):1976-82 DOI: http://dx.doi.org/10.1097/J.PAIN.0000000000001939.

Salomons T V., Moayedi M, Weissman-Fogel I, Goldberg MB, Freeman B V., Tenenbaum HC, Davis KD. Perceived helplessness is associated with individual differences in the central motor output system. Eur J Neurosci [Internet]. Eur J Neurosci; 2012 [cited 2020 Nov 2];35(9):1481-7 DOI: http://dx.doi.org/10.1111/j.1460-9568.2012.08048.x.

Available

from: https://pubmed.ncbi.nlm.nih.gov/22564074/

Simmonds MJ, Moseley GL, Vlaeyen JWS. Pain, mind, and movement: an expanded, updated, and integrated conceptualization. Clin J Pain. 2008;24(4):279-80 DOI: http://dx.doi.org/10.1097/AJP.0b013e31815b607e.

Sullivan MJL, Thibault P, Andrikonyte J, Butler H, Catchlove R, Larivière C. Psychological influences on repetition-induced summation of activity-related pain in patients with chronic low back pain. Pain. 2009;141(1-2):70-8 DOI: http://dx.doi.org/10.1016/J.PAIN.2008.10.017.

Tiemann L, Hohn VD, Ta Dinh S, May ES, Nickel MM, Gross J, Ploner M. Distinct patterns of brain activity mediate perceptual and motor and autonomic responses to noxious stimuli. Nat Commun. 2018;9(1) DOI: http://dx.doi.org/10.1038/S41467-018-06875-X.

La Touche R, Grande-Alonso M, Arnés-Prieto P, Paris-Alemany A. How does self-efficacy influence pain perception, postural stability and range of motion in individuals with chronic low back pain? Pain Physician. 2019;22(1):E1-13.

Turner J, Arendt-Nielsen L. Four decades later: what's new, what's not in our understanding of pain. Pain. 2020;161(9):1943-4 DOI: http://dx.doi.org/10.1097/J.PAIN.0000000000001991.

Wager TD, Atlas LY, Lindquist MA, Roy M, Woo C-W, Kross E. An fMRI-Based Neurologic Signature of Physical Pain. N Engl J Med. 2013;368(15):1388 DOI: 
http://dx.doi.org/10.1056/NEJMOA1204471.

Wei-Ju C, Neil E O, Beckenkamp PR, Alhassani G, Liston MB, Schabrun SM. Altered Primary Motor Cortex Structure, Organization, and Function in Chronic Pain: A Systematic Review and Meta-Analysis. J Pain. 2018;19(4):341-59 DOI: http://dx.doi.org/10.1016/J.JPAIN.2017.10.007.

Yeater T, Clark D, Hoyos L, Valdes-Hernandez P, Peraza J, Allen K, Cruz-Almeida Y. Chronic Pain is Associated With Reduced Sympathetic Nervous System Reactivity During Simple and Complex Walking Tasks: Potential Cerebral Mechanisms. Chronic Stress (Thousand Oaks, Calif). 2021;5

DOI:

http://dx.doi.org/10.1177/24705470211030273. 